PROCEEDINGS OF THE

AMERICAN MATHEMATICAL SOCIETY

Volume 125, Number 6, June 1997, Pages 1651-1658

S 0002-9939(97)03849-5

\title{
ISOMORPHISMS OF ROW AND COLUMN FINITE MATRIX RINGS
}

\author{
J. HAEFNER, A. DEL RÍO, AND J. J. SIMÓN
}

(Communicated by Ken Goodearl)

\begin{abstract}
This paper investigates the ring-theoretic similarities and the categorical dissimilarities between the ring $R F M(R)$ of row finite matrices and the ring $R C F M(R)$ of row and column finite matrices. For example, we prove that two rings $R$ and $S$ are Morita equivalent if and only if the rings $R C F M(R)$ and $\operatorname{RCFM}(S)$ are isomorphic. This resembles the result of V. P. Camillo (1984) for $R F M(R)$. We also show that the Picard groups of $R F M(R)$ and $R C F M(R)$ are isomorphic, even though the rings $R F M(R)$ and $R C F M(R)$ are never Morita equivalent.
\end{abstract}

\section{INTRODUCTION}

Let $R$ be a ring with identity, let $R F M(R)$ be the ring of row-finite matrices over $R$, let $R C F M(R)$ be the ring of row and column-finite matrices over $R$, let $F C(R)$ be the ring of matrices with a finite number of nonzero columns, and let $F M(R)$ be the ring of all matrices with only a finite number of nonzero entries. All matrices are considered countably indexed. The theme of this paper is that while the rings $R F M(R)$ and $R C F M(R)$ are categorically quite different, they share many ring-theoretic properties. For example, Camillo ([3]) has shown that rings $R$ and $S$ are Morita equivalent if and only if $\operatorname{RFM}(R)$ and $\operatorname{RFM}(S)$ are isomorphic. We prove

Theorem A. Rings $R$ and $S$, with identity, are Morita equivalent if and only if $R C F M(R)$ and $R C F M(S)$ are isomorphic.

Similar to Camillo's proof, the key argument in the proof of Theorem A involves understanding the isomorphisms between $R C F M(R)$ and $R C F M(S)$. Consequently, we also prove

Proposition B. Every (ring) isomorphism between $R C F M(R)$ and $R C F M(S)$ restricts to an isomorphism between $F M(R)$ and $F M(S)$.

Proposition B is fundamental in the study of the Picard groups of $R C F M(R)$ and $R$ and, again, we find a ring-theoretic similarity between $R C F M(R)$ and $R F M(R)$. For example, in [2], the authors show that the Picard group of $R$ is isomorphic to the Picard group of $\operatorname{RFM}(R)$. We prove

Received by the editors January 8, 1996 .

1991 Mathematics Subject Classification. Primary 16D30, 16S50, 16W20.

This paper was written while the first author was visiting the Universidad de Murcia with a grant from DGICYT (SAB 95-0215).

The second and third authors have been supported by DGICYT (PB-0300-C02-02). 
Theorem C. The Picard group of $R F M(R)$ is isomorphic to the Picard group of $\operatorname{RCFM}(R)$.

To prove this theorem, we first show that an automorphism of $R F M(R)$ is the product of an inner automorphism and an automorphism that restricts to an automorphism of $F M(R)$.

Finally, while the rings $R F M(R)$ and $R C F M(R)$ share ring-theoretic properties as seen in the above results, our last theorem shows that they are very different in a categorical sense. We prove

Theorem D. There do not exist rings $R$ and $S$ (with identity) such that $R F M(S)$ is Morita equivalent to $\operatorname{RCFM}(R)$.

So, for example, although the Picard groups of $R F M(R)$ and $R C F M(R)$ are isomorphic, the rings themselves are not Morita equivalent.

The main tool for all these applications appears in section 3 , in which we prove that $F M(R)$ is the largest 2-sided ideal in $R C F M(R)$ satisfying some technical property. See Lemma 1. Section 4 is devoted towards proving our above-mentioned results.

\section{Notation AND PRELIMINARIES}

Let $R$ be a ring with identity. We will write the action of homomorphisms of left modules on the right.

For every $i, j \in \mathbf{N}, e_{i j} \in F M(R)$ is the basic matrix having $1 \in R$ in the $i j$-place and zero in each other. We denote $e_{i}=e_{i i}$. For any finite subset $X \subset \mathbf{N}$ we denote $e_{X}=\sum_{x \in X} e_{x}$. For any matrix $\alpha$ and $i, j \in \mathbf{N}, \alpha(i, j)$ denotes the $(i, j)$-entry of $\alpha$.

The following facts will be used without explicit mention. $F C(R)$ is a two-sided ideal of $\operatorname{RFM}(R)$ and it is generated by $\left\{e_{i} \mid i \in \mathbf{N}\right\}$ as a left ideal. $F M(R)$ is a two-sided ideal of $\operatorname{RCFM}(R)$ and it is generated by $\left\{e_{i} \mid i \in \mathbf{N}\right\}$ both as left ideal and as right ideal. Actually, $R C F M(R)$ is the idealizer of $F M(R)$ in $R F M(R)$. $F M(R)$ is the right ideal of $R F M(R)$ generated by $\left\{e_{i} \mid i \in \mathbf{N}\right\}$ (see[5]). Moreover,

$$
\begin{aligned}
F M(R) & =\bigcup_{\substack{X \subset \mathbb{N} \\
X \text { finite }}} e_{X} \operatorname{RFM}(R) \\
& =\bigcup_{\substack{X \subset \mathbb{N} \\
X \text { finite }}} R C F M(R) e_{X}=\bigcup_{\substack{X \subset \mathbb{N} \\
X \text { finite }}} e_{X} \operatorname{RCFM} M(R)
\end{aligned}
$$

and

$$
F C(R)=\bigcup_{\substack{X \subset \mathrm{N} \\ X \text { finite }}} R F M(R) e_{X} .
$$

We observe that if $f \in R F M(R)$ and $f e_{i}=0$ for all $i \in \mathbf{N}$, then $f=0$ and the symmetric property also holds.

Finally, $R F M(R)$ is isomorphic to $\operatorname{End}\left({ }_{R} R^{(\mathbf{N})}\right)$ and to $\operatorname{End}(F M(R) F M(R))$ (by right multiplications).

\section{The Fundamental Lemma}

To prove the results posed in the introduction, we first show that $F M(R)$ is the largest two-sided ideal of $R C F M(R)$ in a certain sense. 
Lemma 1. Let $R$ be a ring with identity and suppose that there exists a family $\left\{f_{i j}\right\}_{i j \in \mathbf{N}}$ of nonzero elements of $R C F M(R)$ such that:

1. $f_{i j} f_{k l}=\delta_{j k} f_{i l}$, for every $i, j, k, l \in \mathbf{N}$.

2. $J=\sum_{\mathbf{N}} R C F M(R) f_{i}$ is a two-sided ideal in $R C F M(R)$ (where $\left.f_{i}=f_{i i}\right)$.

Then $J \subseteq F M(R)$.

Proof. Set $I=F M(R)$. If $f_{i j} \in I$ for some $i, j$ then $f_{k l}=f_{k i} f_{i j} f_{j l} \in I$, for every $k, l \in \mathbf{N}$ (because $I$ is a two-sided ideal of $A$ ) and hence $J \subseteq I$. Therefore one may assume that $f_{i j} \notin I$ for all $i, j \in \mathbf{N}$.

For each $n \in \mathbf{N}$ set $X_{n}=\left\{i \in \mathbf{N} \mid f_{1} e_{i} f_{1 n} \neq 0\right\}$. We claim that $X_{n} \neq \emptyset$. To see this, first note that $f_{1} f_{1 n}=f_{1 n} \neq 0$ and so there is an $i \in \mathbf{N}$ such that $0 \neq e_{i} f_{1} \in I$. Thus $e_{i} f_{1}=e_{i} f_{1} e_{X}$, for some finite subset $X \subset \mathbf{N}$ and hence $0 \neq e_{i} f_{1 n}=e_{i} f_{1} f_{1 n}=e_{i} f_{1} e_{X} f_{1 n}=\sum_{x \in X} e_{i} f_{1} e_{x} f_{1 n}$ so that there is an $x \in X$ such that $f_{1} e_{x} f_{1 n} \neq 0$.

Next we prove that $X_{n}$ is an infinite set. Suppose $X_{n}$ is finite. For every $k \in \mathbf{N}$, let $P_{k}=\left\{r \in \mathbf{N} \mid e_{k} f_{1} e_{r} \neq 0\right\}$. Then $e_{k} f_{1 n}=e_{k} f_{1} e_{P_{k}} f_{1 n}=e_{k} f_{1} e_{P_{k} \cap X_{n}} f_{1 n}=$ $e_{k} f_{1} e_{X_{n}} f_{1 n}$, for every $k \in \mathbf{N}$ and hence $f_{1 n}=f_{1} e_{X_{n}} f_{1 n} \in I$ which contradicts our assumption.

We recursively construct two sequences, $\left(i_{j}\right)_{j \in \mathbf{N}}$ and $\left(k_{j}\right)_{j \in \mathbf{N}}$, of natural numbers such that the first sequence consists of elements from $X_{n}$, while second one is strictly increasing. This will ultimately generate a contradiction to our assumption that $f_{i j} \notin I$ for all $i, j \in \mathbf{N}$.

Let $i_{1}$ be the first element of $X_{1}, Z_{1}=\left\{r \in \mathbf{N} \mid e_{r} f_{1} e_{i_{1}} \neq 0\right.$ or $\left.e_{i_{1}} f_{1} e_{r} \neq 0\right\}$ and $k_{1}=\max Z_{1}$. For every $n>1$, let

$$
Y_{n}=\left\{r \in \mathbf{N} \mid e_{m} f_{1} e_{r} \neq 0 \text { or } e_{r} f_{1 n} e_{m} \neq 0 \text { for some } m \leq k_{n-1}\right\} .
$$

It is clear that $Y_{n}$ is a finite set. Now we define $i_{n}$ to be the first element of $X_{n}-Y_{n}$ and $Z_{n}=\left\{r \in \mathbf{N} \mid e_{r} f_{1} e_{i_{n}} \neq 0\right.$ or $\left.e_{i_{n}} f_{1 n} e_{r} \neq 0\right\}$. Note that $Z_{n}$ is a finite set and is not empty because $i_{n} \in X_{n}$. Further since $i_{n} \notin Y_{n}, r>k_{n-1}$ for every $r \in Z_{n}$. In particular $k_{n}=\max Z_{n}>k_{n-1}$.

Let $\alpha$ be the $\mathbf{N} \times \mathbf{N}$ matrix over $R$ given by

$$
\alpha(i, j)= \begin{cases}\left(f_{1} e_{i_{n}} f_{1 n}\right)(i, j) & \text { if } k_{n-1}<i, j \leq k_{n} \text { for some } n \in \mathbf{N}, \\ 0 & \text { otherwise. }\end{cases}
$$

Obviously $\alpha \in R C F M(R)$. Let $K_{n}=\left\{i \in \mathbf{N} \mid k_{n-1}<i \leq k_{n}\right\}$. Note that $e_{K_{n}} f_{1} e_{i_{n}} f_{1 n}=f_{1} e_{i_{n}} f_{1 n} e_{K_{n}}=f_{1} e_{i_{n}} f_{1 n}$, because if $e_{x} f_{1} e_{i_{n}} f_{1 n} \neq 0$ then $x \in Z_{n} \subseteq$ $K_{n}$ and similarly $f_{1} e_{i_{n}} f_{1 n} e_{x} \neq 0$ implies that $x \in Z_{n} \subseteq K_{n}$. Hence we have that $e_{K_{n}} \alpha=\alpha e_{K_{n}}=f_{1} e_{i_{n}} f_{1 n}$ for every $n \in \mathbf{N}$.

Now we show two properties of $\alpha$. First, we assert that $\alpha=f_{1} \alpha$. Indeed, if $j \in \mathbf{N}$, then $j \in K_{n}$ for some $n$. Therefore $\alpha e_{j}=\alpha e_{K_{n}} e_{j}=f_{1} e_{i_{n}} f_{1 n} e_{j}=f_{1} \cdot f_{1} e_{i_{n}} f_{1 n} e_{j}=$ $f_{1} \alpha e_{K_{n}} e_{j}=f_{1} \alpha e_{j}$. We conclude that $\alpha=f_{1} \alpha$.

Second, we assert that $\alpha f_{n} \neq 0$ for all $n \in \mathbf{N}$. To see this, note that $e_{K_{n}} \alpha f_{n}=$ $f_{1} e_{i_{n}} f_{1 n} f_{n}=f_{1} e_{i_{n}} f_{1 n} \neq 0$. Thus $\alpha f_{n} \neq 0$ for all $n \in \mathbf{N}$.

But as $\alpha \in R C F M(R), f_{1} \alpha \in f_{1} R C F M(R) \subseteq J$ because $J$ is two-sided. Therefore $f_{1} \alpha=\sum_{j \in F} f_{1} \alpha f_{j}$, where $F$ is a finite subset of $\mathbf{N}$, so that $f_{1} \alpha f_{n}=0$ for almost all $n \in \mathbf{N}$, which contradicts the second property of $\alpha$.

It is obvious that condition 1 of Proposition 1 cannot be deleted. The following example shows that condition 2 of Proposition 1 is also not superfluous. 
Example 2. There exist a ring $R$, a family $\left\{f_{i j}\right\}_{i j \in \mathbf{N}} \subseteq R C F M(R)$ such that $f_{i j} f_{k l}=\delta_{j k} f_{i l}$ for every $i, j, k, l \in \mathbf{N}$, and a left ideal, $J=\sum R C F M(R) f_{i}$, that properly contains $F M(R)$.

Proof. Let $\beta: \mathbf{N} \rightarrow \mathbf{N}^{2}$ be a bijection. This bijection induces an isomorphism of $R$-bimodules $\beta:{ }_{R} R_{R}^{(\mathbf{N})} \rightarrow{ }_{R}\left(R^{(\mathbf{N})}\right)_{R}^{(\mathbf{N})}$ which induces two ring isomorphisms $\beta_{l}: \operatorname{End}\left({ }_{R} R^{(\mathbf{N})}\right) \rightarrow \operatorname{End}\left({ }_{R}\left(R^{(\mathbf{N})}\right)^{(\mathbf{N})}\right)$ and $\beta_{r}: \operatorname{End}\left(R_{R}^{(\mathbf{N})}\right) \rightarrow \operatorname{End}\left(\left(R^{(\mathbf{N})}\right)_{R}^{(\mathbf{N})}\right)$. We recall that $\operatorname{End}\left({ }_{R}\left(R^{(\mathbf{N})}\right)^{(\mathbf{N})}\right)$ is isomorphic to the ring $R C M(R F M(R))$ of row-convergent matrices over $R F M(R)$ and $\operatorname{End}\left(\left(R^{(\mathbf{N})}\right)_{R}^{(\mathbf{N})}\right)$ is isomorphic to the ring $C C M(C F M(R))$ of column convergent matrices over $C F M(R)$ ([4, Theorem 106.1]). Now having in mind the nature of these isomorphisms, one can check they induce an isomorphism $\sigma$ between $R C F M(R)$ and $R C C M(R C F M(R))=$ $R C M(R F M(R)) \cap C C M(C F M(R))$. Further, one can check that $\sigma(F M(R))=$ $F M(F M(R))$.

Let $K=R C C M(R C F M(R))$ and $f_{i j}^{\prime} \in K$ such that $f_{i j}^{\prime}$ has $1 \in R C F M(R)$ in the $i j$-place and zero elsewhere. Let $J=\sum K f_{i i}^{\prime}$. Clearly, $F M(F M(R))$ is contained properly in $J$ and taking $f_{i j}=\sigma^{-1}\left(f_{i j}\right)$ we have the desired family. We show explicitly that $J$ is not a two-sided ideal. Take $x \in K$ such that $x$ has $e_{1 j} \in F M(R)$ in the $1 j$-entry and zero elsewhere. Since $J \subseteq R F M(R F M(R))$, $x \notin J$. But, $f_{11} e_{1 j}=e_{1 j}$ for every $j \in \mathbf{N}$, and hence $x=f_{11} x$.

\section{Comparing $R F M(R)$ with $R C F M(R)$}

In this section, we prove the results mentioned in the introduction. We begin with Theorem A and Proposition B. The key to the main result of [3] is, in essence, that an isomorphism $\phi: R F M(R) \rightarrow R F M(S)$ satisfies $\phi(F C(R))=F C(S)$, where $F C$ is the ring of matrices with only finitely many non-zero columns. In our setting, with $R C F M(R)$ taking the place of $R F M(R)$, this result translates into Proposition $\mathrm{B}$ from the Introduction.

Proposition 3. Let $R$ and $S$ be any two rings with identity.

(a) Every ring isomorphism $\delta: R C F M(R) \rightarrow R C F M(S)$ satisfies

$$
\delta(F M(R))=F M(S) .
$$

(b) Every ring isomorphism $\delta: R C F M(R) \rightarrow R C F M(S)$ extends, in a unique way, to an isomorphism $\delta^{\prime}: R F M(R) \rightarrow R F M(S)$.

(c) Every ring isomorphism $\sigma: R F M(R) \rightarrow R F M(S)$ such that

$$
\sigma(F M(R))=F M(S)
$$

satisfies $\sigma(R C F M(R))=R C F M(S)$.

(d) There is a group monomorphism

$$
\phi: \operatorname{Aut}(R C F M(R)) \rightarrow \operatorname{Aut}(R F M(R))
$$

via $\phi(\delta)=\delta^{\prime}$ using (b) above. Moreover, the image of $\phi$ is the subgroup of automorphisms of $R F M(R)$ that restrict to automorphisms of $F M(R)$.

Proof. (a) follows immediately from Lemma 1. Using the fact that $R F M(R)$ is isomorphic to $\operatorname{End}\left({ }_{F M(R)} F M(R)\right)$, (b) is straightforward. We get (c) from the fact that $R C F M(R)$ is the idealizer of $F M(R)$ in $R F M(R)$. Finally, (d) follows from (a), (b), and (c).

Now we can prove an analogue to Camillo's result for $R C F M(R)$. 
Theorem 4. Let $R$ and $S$ be rings with identity. $R$ and $S$ are Morita equivalent rings if and only if $R C F M(R)$ and $R C F M(S)$ are isomorphic rings.

Proof. Assume first that $R$ and $S$ are Morita equivalent rings. Let ${ }_{R} P$ be a progenerator such that $\operatorname{End}\left({ }_{R} P\right) \cong S$ as rings. By [1, Lemma 1.2] we have that there exists a ring isomorphism $\alpha^{*}: R F M(R) \rightarrow R F M\left(\operatorname{End}\left({ }_{R} P\right)\right)$ such that $\alpha^{*}(F M(R))=$ $F M\left(\operatorname{End}\left({ }_{R} P\right)\right)$. Let $\beta: R F M\left(\operatorname{End}\left({ }_{R} P\right)\right) \rightarrow R F M(S)$ be induced (coordinatewise) by the isomorphism $\operatorname{End}\left({ }_{R} P\right) \cong S$, and let $\delta=\beta \circ \alpha^{*}$. Then it is clear that $\delta(F M(R))=F M(S)$. Since $R C F M(R)$ (resp. $R C F M(S))$ is the idealizer of $F M(R)($ resp. $F M(S)), \delta(R C F M(R))=R C F M(S)$. $1)]$.

The converse follows from Proposition 3 together with [1, Theorem 2.5 (3 implies

It is interesting to note that there are some rings between $F M(R)$ and $R C F M(R)$ which have automorphisms that do not restrict to automorphisms of $F M(R)$, as the next example shows.

Example 5. There exist rings with identity, $R$ and $S$, such that $F M(R) \subset S \subset$ $R C F M(R)$ and an automorphism of $S$, say $\delta$, such that $\delta(F M(R)) \neq F M(R)$.

Proof. Let $K$ be any ring with identity and let $B=\operatorname{RCFM}(\operatorname{RCFM}(K))$. As we saw in Example 2 there exists an injective ring homomorphism $\beta: B \rightarrow R C F M(K)$. Let $T$ be the image of $\beta$, let $R=K \times R C F M(K)$, and let $S=T \times B$. After identifying $R C F M(R)$ with $R C F M(K) \times B$, it is clear that $F M(R) \subset S \subset R C F M(R)$ and that $\delta: S \rightarrow S$ via $\delta(x, y)=\left(\beta(y), \beta^{-1}(x)\right)$ is an automorphism of $S$. But an straightforward calculation shows that $\delta(F M(R)) \neq F M(R)$.

The ring $R F M(R)$ is another ring for which there are automorphisms of $R F M(R)$ that do not restrict to automorphisms of $F M(R)$; see [1]. Nonetheless, we show that these pathological automorphisms are "controlled" by the inner automorphisms of $R F M(R)$. In particular, we show that every automorphism of $R F M(R)$ is a product of an inner automorphism and an automorphism that restricts to an automorphism of $F M(R)$.

Proposition 6. For every $\sigma \in \operatorname{Aut}(R F M(R))$, there exists $\tau \in \operatorname{Inn}(R F M(R))$ such that $\sigma \tau(F M(R))=F M(R)$.

Proof. Let $\sigma \in \operatorname{Aut}(\operatorname{RFM}(R))$. Then $P=R^{(\mathbf{N})} \sigma\left(e_{1}\right)$ is a progenerator as left $R$-module such that $\operatorname{End}\left({ }_{R} P\right)$ is isomorphic to $R$ [3]. Specifically, the isomorphism $\tau: R \rightarrow \operatorname{End}\left({ }_{R} P\right)$ is given by $(p) \tau(r)=p \sigma\left(e_{1} D(r)\right)$ where $D(r)$ denotes the scalar matrix defined by $r$. We consider $P$ as an $R$-bimodule using this isomorphism; explicitly, $r \cdot p \cdot s=r p \tau(s)(r, s \in R, p \in P)$. Define $\tau^{*}: R F M(R) \rightarrow R F M(\operatorname{End}(P))$ via a coordinate-wise application of $\tau$.

The map $f: P^{(\mathbf{N})} \rightarrow R^{(\mathbf{N})}$ given by $\left(\left(p_{i}\right)_{i \in \mathbf{N}}\right) f=\sum_{i \in \mathbf{N}} p_{i} \sigma\left(e_{1 i}\right)$ is an isomorphism whose inverse is given by $\left(\left(r_{i}\right)_{i \in \mathbf{N}}\right) f^{-1}=\left(r_{i} \sigma\left(e_{i 1}\right)\right)_{i \in \mathbf{N}}$.

We identify $R F M(R)$ with $\operatorname{End}\left(R^{(\mathbf{N})}\right)$ and $R F M(\operatorname{End}(P))$ with $\operatorname{End}\left(P^{(\mathbf{N})}\right)$ canonically. For every $x \in R F M(R)$, the following diagram is commutative:

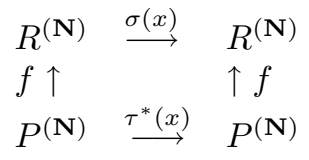


To see this, observe

$$
\begin{aligned}
(p) \tau^{*}(x) f & =\left(\left(\sum_{i \in \mathbf{N}} p_{i} \tau\left(x_{i j}\right)\right)_{j \in \mathbf{N}}\right) f \\
& =\left(\left(\sum_{i \in \mathbf{N}} p_{i} \sigma\left(e_{1} D\left(x_{i j}\right)\right)\right)_{j \in \mathbf{N}}\right) f \\
& =\sum_{j \in \mathbf{N}} \sum_{i \in \mathbf{N}} p_{i} \sigma\left(e_{1} D\left(x_{i j}\right) e_{1 j}\right) \\
& =\sum_{i \in \mathbf{N}} p_{i} \sigma\left(e_{1} \sum_{j \in \mathbf{N}} D\left(x_{i j}\right) e_{1 j}\right) \\
& =\sum_{i \in \mathbf{N}} p_{i} \sigma\left(e_{1 i} x\right) \\
& =(p) f \sigma(x) .
\end{aligned}
$$

Now let $\alpha: P^{(\mathbf{N})} \rightarrow R^{(\mathbf{N})}$ be the isomorphism mentioned in [1], which induces an isomorphism $\alpha^{*}: R F M(\operatorname{End}(P))=\operatorname{End}\left(P^{(\mathbf{N})}\right) \rightarrow \operatorname{End}\left(R^{(\mathbf{N})}\right)=R F M(R)$ such that $\alpha^{*}\left(F M\left(\operatorname{End}\left({ }_{R} P\right)\right)\right)=F M(R)$. More concretely, $\alpha^{*}$ is characterized by the property that, for every $y \in \operatorname{End}\left(P^{(\mathbf{N})}\right)$, the following diagram is commutative:

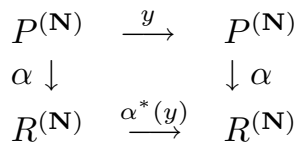

Therefore, the following diagram is commutative, for every $x \in R F M(R)$ :

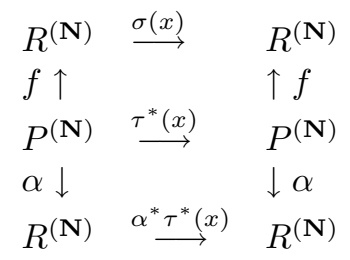

It follows that $\sigma^{-1} \alpha^{*} \tau^{*}$ is the inner automorphism of $R F M(R)$ induced by $\alpha^{-1} f$ and $\alpha^{*} \tau^{*}(F M(R))=F M(R)$.

Recall that the Picard group of a ring $T$ is the multiplicative group consisting of the bimodule isomorphism classes of invertible $T$-bimodules. We now prove Theorem C from the Introduction.

Theorem 7. For every ring $R$,

$$
\operatorname{Pic}(R) \simeq \operatorname{Pic}(R F M(R)) \simeq \operatorname{Pic}(F M(R)) \simeq \operatorname{Pic}(R C F M(R)) .
$$

Proof. It has been shown in [2] that $\operatorname{Pic}(R) \simeq \operatorname{Pic}(R F M(R)) \simeq \operatorname{Pic}(F M(R))$. On the other hand, both $R F M(R)$ and $R C F M(R)$ have the SBN property, so that $\operatorname{Pic}(R C F M(R))=\operatorname{Out}(R C F M(R))$ and $\operatorname{Pic}(R F M(R))=\operatorname{Out}(R F M(R))$; see [2]. Thus, it suffices to show that $\operatorname{Out}(R C F M(R)) \simeq \operatorname{Out}(R F M(R))$.

From Proposition 3, there is a group monomorphism $\phi: \operatorname{Aut}(R C F M(R)) \rightarrow$ $\operatorname{Aut}(R F M(R))$ such that the image of $\phi$ is the subgroup of automorphisms of $R F M(R)$ that restrict to automorphisms of $F M(R)$. In particular, $\phi(\delta)=\delta^{\prime}$ using (b) of Proposition 3. We claim that

$$
\phi(\operatorname{Inn}(R C F M(R)))=\operatorname{Inn}(R F M(R)) \cap \operatorname{Im}(\phi) .
$$

It is clear that $\phi(\operatorname{Inn}(R C F M(R))) \subseteq \operatorname{Inn}(R F M(R))$. For the opposite inclusion, note that if $\sigma \in \operatorname{Aut}(R C F M(R))$ such that $\phi(\sigma) \in \operatorname{Inn}(R F M(R))$, then there exists a unit $u \in R F M(R)$ for which $u F M(R)=F M(R) u$. In particular, for each $i, u \cdot e_{i i} \in F M(R)$ so that $e_{j j} \cdot u \cdot e_{i i}=0$ for almost all values of $j$. Hence, 
$u \in R C F M(R)$ and so $\sigma \in \operatorname{Inn}(R C F M(R))$. This completes our claim. Therefore, $\phi$ induces an isomorphism between $\operatorname{Out}(R C F M(R))$ and

$$
\frac{\operatorname{Im}(\phi) \cdot \operatorname{Inn}(R F M(R))}{\operatorname{Inn}(R F M(R))} .
$$

By Proposition 6, the above quotient module is isomorphic to $\operatorname{Out}(R F M(R))$.

While the previous results show that the rings $R F M(R)$ and $R C F M(S)$ share many ring-theoretical properties, they are quite different categorically. We conclude this paper with our proof of Theorem D.

Theorem 8. For any two rings with identity, $R$ and $S$ the rings $R F M(R)$ and $R C F M(S)$ cannot be Morita equivalent. In particular, they are not isomorphic.

Proof. Let $E=R F M(R), B=R C F M(S), I=F C(R)$, and $J=F M(S)$.

Assume that $E$ and $B$ are Morita equivalent rings. Then, by [6] we have that there exists a natural number $n \in \mathbf{N}$ such that $E$ and $\mathbf{M}_{n}(B)$ are isomorphic rings. But $B$ and $\mathbf{M}_{n}(B)$ are isomorphic. Indeed, the map $\alpha: B \rightarrow \mathbf{M}_{n}(B)$ given by $\alpha(X)(i, j)(a, b)=X(n(a-1)+i, n(b-1)+j)(X \in B, 1 \leq i, j \leq n, a, b \in \mathbf{N})$ is a ring isomorphism.

Let $\delta: E \rightarrow B$ be a ring isomorphism, let $\left\{e_{i j}\right\}_{i j \in \mathbf{N}}$ and $\left\{f_{i j}\right\}_{i j \in \mathbf{N}}$ be the basic matrices of $E$ and $B$, respectively, and let $e_{i j}^{\prime}=\delta\left(e_{i j}\right)$ and $f_{i j}^{\prime}=\delta^{-1}\left(f_{i j}\right)$.

We show that $I=\delta^{-1}(J)$. Since $I=\sum_{\mathbf{N}} E e_{i}$ is a two-sided ideal of $E$, we have that $\delta(I)=\sum_{\mathbf{N}} B e_{i}^{\prime}$ is a two-sided ideal of $B$ and the family $\left\{e_{i j}^{\prime}\right\}$ verifies the conditions of Lemma 1 . We conclude that $\delta(I) \subseteq J$ and so $I \subseteq \delta^{-1}(J)$. Consequently, $I=\oplus I f_{i}^{\prime}$.

Now we use analogous ideas to those found in [3]. Let $\alpha: I f_{1}^{\prime} \rightarrow \sum_{\mathbf{N}} I f_{i}^{\prime}=I$ be any $E$-homomorphism. Then there exists $\bar{\alpha}: I \rightarrow I$ such that $\alpha=\bar{\alpha} \circ f_{1}^{\prime}$. By [5], $\bar{\alpha}$ is the right multiplication by some $a \in E$. It is clear that $a \in f_{1}^{\prime} J$ and hence $\delta(a) \in f_{1} B$. Therefore, $\delta(a)=\delta(a) \sum_{\text {finite }} f_{j}$ and hence $a=a \sum_{\text {finite }} f_{j}^{\prime}$. Thus $\alpha\left(I f_{1}^{\prime}\right) \subseteq \bigoplus \sum_{\text {finite }} I f_{i}^{\prime}$. Since ${ }_{E} I f_{1}^{\prime} \simeq{ }_{E} I f_{i}^{\prime}$, for every $i \in \mathbf{N}$, we use [3] to conclude that ${ }_{E} I f_{1}^{\prime}$ must be finitely generated. Let $x_{1} f_{1}^{\prime}, \ldots, x_{n} f_{1}^{\prime}$ be a family of generators of ${ }_{E} I f_{1}^{\prime}$ with $x_{i} \in I$. Then $I f_{1}^{\prime}=\sum_{i=1}^{n} E x_{i} f_{1}^{\prime}$, and hence there is a finite subset $F$ of $\mathbf{N}$, such that $I f_{1}^{\prime} \subseteq E e_{F}$. This implies that $e_{i} f_{1}^{\prime}=e_{i} f_{1}^{\prime} e_{F}$ for every $i \in \mathbf{N}$, and hence $f_{1}^{\prime}=f_{1}^{\prime} e_{F} \in I$. Thus $f_{i}^{\prime}=f_{i 1}^{\prime} f_{1}^{\prime} f_{1 i}^{\prime} \in I$ for every $i \in \mathbf{N}$ and we conclude that $\delta^{-1}(J) \subseteq I$.

To finish the proof, let $x \in R F M(S)-B$, and let $\rho_{x}$ denote right multiplication by $x$. We have the homomorphism $\delta\left(\rho_{x}\right) \delta^{-1}:_{B} I \rightarrow_{B} I$, and there exists $y \in$ $R F M(R)$ such that $\delta\left(\rho_{x}\right) \delta^{-1}=\rho_{y}$. For every $a \in I, a(y) \delta=\left((a) \delta^{-1} y\right) \delta=a x$. Therefore, $\delta(y)=x$ contradicting the fact that $x \notin B$. This finishes the proof.

\section{REFERENCES}

[1] G.D. Abrams, Infinite matrix types which determine Morita equivalence. Arch. Math. ( 46), 1986, 33-37. MR 87f:16015

[2] G. Abrams and J. Haefner, Picard groups and infinite matrix rings. Trans. Amer. Math. Soc., to appear.

[3] V.P. Camillo, Morita equivalence and infinite matrix rings. Proc. Amer. Math. Soc. (2)( 90), 1984, 186-188. MR 85a:16045

[4] L. Fuchs, Infinite abelian groups, Vol. II, Acad. Press, New York, 1973. MR 50:2362 
[5] J.L. García, The Characterization problem for endomorphism rings. J. Austral Math. Soc. (Series A) ( 50) 1991, 116-137. MR 92a:16035

[6] J. J. Simón, Morita equivalent row finite matrix rings are isomorphic, J. Algebra, ( 173) 1995, 390-393. MR 96d:16012

Department of Mathematics, University of Colorado, Colorado Springs, Colorado 80933

E-mail address: haefner@math.uccs.edu

Departamento de Matemáticas, Universidad de Murcia, 30071, Murcia, Spain

E-mail address: adelrio@fcu.um.es

E-mail address: jsimon@fcu.um.es 